\title{
Schützt moderater Weinkonsum vor nichtalkoholischer Fettleber?
}

\author{
Nicolai Worm \\ München, Germany
}

Die nichtalkoholische Fettleber (abgekürzt mit NAFLD vom Englischen Non-Alcoholic Fatty Liver Disease) hat sich zur neuen Volkskrankheit entwickelt [1]. Sie ist charakterisiert durch eine variable Einlagerung von Lipiden in die Hepatozyten. "Fettleber" wird diagnostiziert, wenn mehr als 50\% der Leberzellen sichtbar Fett speichern oder wenn der Fettgehalt mehr als 5,5\% des Lebergewichts ausmacht. Die Fetteinlagerung darf dabei nicht durch erhöhten Alkoholkonsum (bis zu $20 \mathrm{~g}$ pro Tag für Frauen und bis $\mathrm{zu} 30 \mathrm{~g}$ pro Tag für Männer), durch die Einnahme von Arzneimitteln oder durch erbliche Erkrankungen bedingt sein [2].

Der Oberbegriff NAFLD umfasst ein breites morphologisches Spektrum und reicht von der einfachen Steatose über die Steatohepatitis bis zur Leberzirrhose.

Schätzungsweise 30 bis 40 Prozent der Erwachsenen in westlichen Industrieländern sind bereits betroffen $[3,4]$. Bei übergewichtigen Erwachsenen sind es schon etwa 70 Prozent - und besonders bedenklich: Jedes dritte übergewichtige Schulkind hat bereits eine Fettleber [5]! Die Typ-2-Diabetiker weisen sogar zu 80 Prozent eine NAFLD auf $[6,7]$.

Bedenklich ist die NAFLD nicht nur, weil daraus eine chronische Leberentzündung, Leberzirrhose und Leberkrebs entstehen kann [8]. Inzwischen ist geklärt, dass sie als Folge der Insulinresistenz die hepatische Manifestation des metabolischen Syndroms darstellt [9, 10] und die Entwicklung zum Typ-2-Diabetes mellitus in erheblichem Maße fördert $[6,7,11]$. Außerdem weisen immer mehr Studien darauf hin, dass die NAFLD ein von allen bekannten Risikofaktoren unabhängiges Risiko für Herz-Kreislaufkrankheiten darstellt [12].

Die NAFLD ist die Folge modernen Lebensstils $[10,13]$. Zu viel Nahrungsenergie und zu wenig Bewegung gelten als besonders relevante Risikofaktoren. Bekanntlich fördert chronischer Alkoholmissbrauch die Entwicklung einer alkoholischen Fettleber.

Im Gegensatz dazu konnten in jüngerer Zeit eine Reihe von epidemiologischen Querschnittstudien aufzeigen, dass moderater Alkohol- und insbesondere moderater Weinkonsum mit einer niedrigeren Prävalenz von NAFLD einher gehen [14-18]. Erste Langzeitbeobachtungsstudien, die eine wesentlich bessere Aussagekraft als Querschnittsstudien haben, konnten kürzlich ebenfalls eine niedrigere Inzidenz der NAFLD bei moderatem Alkoholbzw. Weinkonsum aufzeigen [19,20].

Als Wirkmechanismen dieses paradox anmutenden Zusammenhangs werden vor allem eine Förderung der Insulinsensitivität und die verstärkte Leberdurchblutung, wie auch die erhöhten Adiponektinspiegel durch moderaten Alkoholkonsum diskutiert [19]. Aber auch die entzündungshemmenden Effekte von Alkohol per se wie auch von Polyphenolen in Wein und anderen alkoholischen Getränken und auch die antioxidativen Wirkungen der Polyphenole könnten eine protektive Rolle spielen. So kann Resveratrol beispielsweise den Transkriptionsfaktor SREBP-1c für die "de novoLipogenese" aus Kohlenhydraten in der Leber hemmen [21].

$\mathrm{Ob}$ und in wie weit moderater Weinkonsum tatsächlich ursächlich der Entstehung von NAFLD entgegen- wirken kann, ist bis heute noch nicht endgültig geklärt und muss noch weiter erforscht werden.

\section{Literatur}

[1] K. Hassan, V. Bhalla, M.E. El Regal and A.K. HH, World J Gastroenterol, 20, 12082 (2014)

[2] H.J. Yoon and B.S. Cha, World J Hepatol, 6, 800 (2014)

[3] C.D. Williams, J. Stengel and M.L. Asike, Gastroenterology, 140, 124 (2011)

[4] M. Lazo, R. Hernaez and M.S. Eberhardt Am J Epidemiol, 178, 38 (2013)

[5] C. Denzer, Bundesgesundheitsblatt Gesundheitsforschung Gesundheitsschutz, 56, 517 (2013)

[6] A. Sima, R. Timar and A. Vlad, Wien Klin Wochenschr, 126, 335 (2014)

[7] S. Zelber-Sagi, R. Lotan and O. Shibolet, Liver Int, 33, 1406 (2013)

[8] G.A. Michelotti, M.V. Machado and A.M. Diehl, Nat Rev Gastroenterol Hepatol, 10, 656 (2013)

[9] G. Targher and C.D. Byrne, Metab Syndr Relat Disord (2015)

[10] H. Yki-Jarvinen, Lancet Diabetes Endocrinol, 2, 901 (2014)

[11] G. Firneisz, World J Gastroenterol, 20, 9072 (2014)

[12] N.N. Than and P.N. Newsome, Atherosclerosis, 239, 192 (2015)

[13] M. Asrih and F.R. Jornayvaz, Mol Cell Endocrinol (2015)

[14] W. Dunn, R. Xu and J.B. Schwimmer, Hepatology, 47, 1947 (2008)

[15] W. Dunn, A.J. Sanyal and E.M. Brunt, J Hepatol, 57, 384 (2012)

[16] M. Hamaguchi, T. Kojima, A. Ohbora, N. Takeda, M. Fukui and T. Kato, World J Gastroenterol, 18, 156 (2012) 
[17] H.K. Kwon, J.K. Greenson and H.S. Conjeevaram, Liver Int, 34, 129 (2014)

[18] M. Sogabe, T. Okahisa and T. Taniguchi, Liver Int (2014)

[19] A. Moriya, Y. Iwasaki and S. Ohguchi, J Hepatol, 62, $921(2015)$
[20] Y. Hashimoto, M. Hamaguchi and T. Kojima, J Gastroenterol Hepatol, 30, 546 (2015)

[21] N. Arias, M.T. Macarulla, L. Aguirre, J. Miranda, M.P. Portillo, J Physiol Biochem, 2015 Apr 1. Epub ahead of print 